Kansas State University Libraries

New Prairie Press

\title{
USING THE BI-LOGISTIC MODEL TO ESTIMATE BODY TEMPERATURE IN FEEDLOT CATTLE
}

M. Kerek

A. M. Parkhurst

T. L. Mader

Follow this and additional works at: https://newprairiepress.org/agstatconference

Part of the Agriculture Commons, and the Applied Statistics Commons

\section{(c) (1) $\Theta(9$}

This work is licensed under a Creative Commons Attribution-Noncommercial-No Derivative Works 4.0 License.

\section{Recommended Citation}

Kerek, M.; Parkhurst, A. M.; and Mader, T. L. (2003). "USING THE BI-LOGISTIC MODEL TO ESTIMATE BODY TEMPERATURE IN FEEDLOT CATTLE," Conference on Applied Statistics in Agriculture. https://doi.org/ $10.4148 / 2475-7772.1186$

This is brought to you for free and open access by the Conferences at New Prairie Press. It has been accepted for inclusion in Conference on Applied Statistics in Agriculture by an authorized administrator of New Prairie Press. For more information, please contact cads@k-state.edu. 


\title{
USING THE BI-LOGISTIC MODEL TO ESTIMATE BODY TEMPERATURE IN FEEDLOT CATTLE
}

\author{
M. Kerek ${ }^{1}$, A. M. Parkhurst ${ }^{1}$, and T. L. Mader $^{2}$ \\ 1. Department of Statistics, University of Nebraska at Lincoln \\ 2. Department of Animal Science, University of Nebraska at Lincoln
}

\begin{abstract}
Processing and handling cattle require an expenditure of energy causing an elevation of body temperature, depending on the ambient conditions. More knowledge of body temperature, $\mathrm{Tb}$, dynamics could lead to more specific recommendations of how far cattle can be moved without stress. The bi-logistic model has been used to describe the handling process. This model estimates several important biological parameters: rate of increase in $\mathrm{Tb}$ (rate of heat challenge), the maximum $\mathrm{Tb}(\max \mathrm{Tb})$, time to reach maximum $\mathrm{Tb}(\mathrm{tmax})$ and recovery rate (rate of decrease in $\mathrm{Tb}$ ). The objectives of this study are: to compare parameter estimates from the bi-logistic model with a segmented version of the model; to investigate the robustness of the model for different definitions of recovery; and, to check for hormetic behavior using switching functions.
\end{abstract}

\section{INTRODUCTION}

Generally, cattle are processed within a few days of coming into the feedlot. There are several benefits of processing feedlot cattle, such as prevention of diseases by inoculation, treatment for parasites, performance enhancement with implants, and overall maintenance of the health of the animals. However, processing could be detrimental if performed during a period of heat stress. For example, on a very hot and humid day in which the panting mechanism cannot eliminate enough heat, processing and handling may create intolerable and even lethal conditions. Therefore, producers have to make sure that processing has more positive than negative effects on the animal, or at least minimize the detrimental aspects. More knowledge of body temperature dynamics could lead to more specific recommendations of when and how far cattle can be moved without stress.

In this study, heat stress (challenge) is defined to be heat produced by moving steers. It is measured as the temperature differential, the difference between the maximum and initial body temperature $(\mathrm{Tb})$. Additional parameters associated with heat production are time to reach maximum $\mathrm{Tb}(\mathrm{tmax})$, challenge rate (rate of increase in $\mathrm{Tb}$ ) and recovery rate (rate of decrease in $\mathrm{Tb}$ ). Some obstacles to analyzing these kinds of data are summarized by Parkhurst and Mader (2000). Among these obstacles are defining the recovery time. Recovery time was defined as the point at which the body temperature returns (if it ever does) to the exact body temperature at the beginning of the move. If this did not occur, an alternative is to identify a lower asymptote. The end time of the asymptote would then represent the recovery time.

There are many models to describe challenge and recovery (Parkhurst and Mader, 2000). Among them is the logistic model, which is an example of limited growth. The relative growth is not constant and is proportional to the amount remaining. 


$$
\frac{\delta Y}{Y \delta X}=\kappa(\alpha-Y)
$$

where $\mathrm{Y}$ is body temperature; $\mathrm{X}$ is time; the asymptote $\alpha$ is the maximum body temperature; and, $\kappa$ is the rate constant.

The solution gives a symmetrical sigmoidal curve with a rate of change which rises to an inflection point then falls.

$$
Y=\frac{\alpha}{1+\beta e^{\kappa X}}
$$

where $\beta$ is the ratio of range (difference between $\alpha$ and the initial value of $Y$ ) to the minimum.

Assuming that challenge and recovery are independent effects and that their combination can be predicted by an additive model, the bi-logistic model is obtained (Parkhurst and Mader, 2000). It is capable of having a maximum and a pair of inflection points.

$$
Y=\frac{\alpha}{1+\beta_{1} e^{\kappa_{1} X}}+\frac{\delta-\alpha}{1+\beta_{2} e^{\kappa_{2} X}}
$$

The above model may be parameterized in terms of the inflection points when $\beta$ is reexpressed in terms of the inflection point, $\tau$, as

$\beta=\exp (-\kappa \tau)$

The resulting inflection point parameterization is then

$$
Y=\frac{\alpha}{1+e^{\kappa(X-\tau)}}
$$

The bi-logistic model may then be written in terms of inflection points.

$$
Y=\frac{\alpha}{1+e^{K_{1}\left(X-\tau_{1}\right)}}+\frac{\delta-\alpha}{1+e^{\kappa_{2}\left(X-\tau_{2}\right)}}+\varepsilon \quad \quad \text { Eq [1] }
$$

where $\alpha$ is the upper asymptote for challenge; $\delta$ is the lower asymptote for recovery which represents the departure from initial body temperature; $\kappa_{1}$ and $\kappa_{2}$ are the rate constants for challenge and recovery, respectively; and $\tau_{1}$ and $\tau_{2}$ are the inflection points for challenge and recovery, respectively.

The shortcomings of the bi-logistic model are that it underestimates the time to reach the maximum body temperature and that it is sensitive to recovery time. The objectives of this study are to compare parameter estimates from the bi-logistic model with a segmented version of the model and to investigate the robustness of the model for different definitions of recovery. We also checked for hormetic behavior using switching functions. 


\section{METHODS}

The temperature data loggers were placed in the left ear of four head of cattle to record the body temperature at two-minute intervals beginning on day 1 . The experimental design is summarized in Table 1. The cattle were moved with as little pressure as possible over the designated distance (200 or $1200 \mathrm{ft}$ ) at a designated day (day 2, 3, 6, and 7) through the processing facility (between 9 and $10 \mathrm{am}$ ) and back to their pens. All steer-day data sets in this study were analyzed but only illustrative examples are presented in the paper.

\section{Segmented Bi-Logistic Model}

A segmented model was obtained using the logistic model for the two segments (challenge and recovery) of the response (Y, body temperature). Different equations were conditionally defined for each segment, depending on whether X (time) is at least (or greater than) tmax, the time at which the maximum body temperature occurs. In order to make the equation smooth and continuous, the two sections are forced to meet at tmax, the joint point.

In other words, both segments have the same response, $Y=\frac{\alpha_{1}}{1+\beta_{1} e^{K_{1} t \max }}+\varepsilon$ at $\mathrm{X}=\mathrm{tmax}$.

If $\mathrm{X} \leq \mathrm{tmax}$ (challenge) $Y=\frac{\alpha_{1}}{1+\beta_{1} e^{\kappa_{1} X}}+\varepsilon$ and

if $\mathrm{X}>\operatorname{tmax}$ (recovery) $Y=\frac{\alpha_{2}}{1+\beta_{2} e^{\kappa_{2}(X-t \max )}}+\varepsilon$ where $\alpha_{2}=\frac{\alpha_{1}}{1+\beta_{1} e^{\kappa_{1} t \max }}$

We assume $\varepsilon \sim \operatorname{iidN}\left(0, \sigma^{2}\right)$.

Two parameterizations of the logistic model were studied in this paper. The inflection point model, described in the introduction, is one parameterization. Another parameterization, or rather a transformation of $\mathrm{X}$, is based on a power of $\mathrm{X}$ and called the power model.

$$
Y=\frac{\alpha}{1+\left(\frac{X}{\tau}\right)^{-\log (-\kappa)}}+\varepsilon
$$

In this study we used inflection point parameterization for challenge and power parameterization for recovery as they showed the best statistical properties (curvature and close-to-linear behavior) for our data.

$$
\text { If } \mathrm{X} \leq \operatorname{tmax} Y=\frac{\alpha_{1}}{1+e^{\kappa\left(X-\tau_{1}\right)}}+\varepsilon \text { and if } \mathrm{X}>\operatorname{tmax} Y=\frac{\alpha_{2}}{1+\left(\frac{X-t \max }{\tau_{2}-t \max }\right)^{-\log \left(-\kappa_{2}\right)}}+\varepsilon \quad \text { Eq.[2] }
$$

where $\alpha_{1}$, is the upper asymptote for challenge; $\alpha_{2}=\frac{\alpha_{1}}{1+e^{\kappa\left(t \max -\tau_{1}\right)}} ; \kappa_{1}$ and $\kappa_{2}$ are the rate constants for challenge and recovery, $\tau_{1}$ and $\tau_{2}$ are the inflection points for challenge and recovery, respectively; tmax is the time at which the maximum body temperature occurs. Note that at $\mathrm{X}=$ tmax both segments reduce to 
$Y=\frac{\alpha_{1}}{1+e^{K\left(t \max -\tau_{1}\right)}}+\varepsilon$

In other words, the two segments are forced to join at $\mathrm{X}=\mathrm{tmax}$.

\section{Hormetic Model}

Hormetic models by definition are non-monotonic (Schabenberger and Birch, 2001). Thus, dose-response models without hormetic effect suggest monotonic changes in the response with increasing or decreasing dosage. Accounting for hormetic effects, Brain and Cousens (1989) add a linear function of $X$ to the asymptote in the numerator and indicate the additional parameter, $\gamma$, represents the initial rate of increase in the response at low levels of X. Switching functions provide a convenient way to add hormetic behavior to a response curve (Schabenberger and Birch, 2001). The switching function (a model without hormetic effect) combined with an additive hormetic component that we present in this paper (hormetic model) is:

$$
Y=\frac{\alpha}{1+e^{\kappa_{1}\left(X-\tau_{1}\right)}}+\frac{(\delta-\alpha)+\gamma X}{1+e^{\kappa_{2}\left(X-\tau_{2}\right)}}+\varepsilon \quad \quad \text { Eq [3] }
$$

where $\alpha$ is the upper asymptote for challenge; $\delta$ is the lower asymptote for recovery which represents the departure from initial body temperature; $\kappa_{1}$ and $\kappa_{2}$ are the rate constants for challenge and recovery, respectively; and $\tau_{1}$ and $\tau_{2}$ are the inflection points for challenge and recovery, respectively; and $\gamma$ is the hormetic effect. When $\gamma=0$, there is no hormetic effect and the hormetic model, Eq. [3], reduces to the bi-logistic model, with inflection point parameterization Eq.[1]. In terms of heat stress, hormetic models adjust for the initial rate of increase in body temperature during the challenge.

Different parameterizations of the switching function (inflection point, power, and combinations with the original) were also tried. Sometimes the functional form of the two switching functions was the same. Other times it was different. These parameterizations either had larger MSEs than those of Eq. [3], did not capture the max Tb quite as well, or some parameters did not show close-to-linear behavior. Some examples of the parameterizations used are:

$$
\begin{aligned}
& Y=\frac{\alpha}{1+\beta e^{\kappa_{1} X}}+\frac{(\delta-\alpha)+\gamma X}{1+\left(\frac{X}{\tau}\right)^{-\log \left(-\kappa_{2}\right)}}, \text { Original-Power Parameterization } \\
& Y=\frac{\alpha}{1+\beta_{1} e^{\kappa_{1} X}}+\frac{(\delta-\alpha)+\gamma X}{1+\beta_{2} e^{\kappa_{2} X}} \text {, Original-Original Parameterization } \\
& Y=\frac{\delta+\gamma X}{1+\beta e^{\kappa X}} \text {, Original: Common } \beta \text { and } \kappa \text { Parameterization }
\end{aligned}
$$




\section{Data Analysis}

SAS (1999) was the tool used for data analysis. For all parameterizations, the MSE and the estimates, SE, CI and skewness for all parameters were found using proc NLIN. Programs were written in proc IML to estimate intrinsic and parameter effects curvature (IN and PE) and close-to-linear behavior (\%Bias and \%excess variation).

\section{Assessing Nonlinear Behavior}

Two assumptions are needed for least squares estimation of parameters in nonlinear regression: planarity and uniformity of coordinates. There are several ways to assess how close a model-data set combination is to satisfying these asymptotic properties. Bates and Watts (1980) proposed relative measures for intrinsic and parameter-effects curvature.

Intrinsic curvature (IN) measures the relative curvature of the expectation surface at the point of convergence. In linear regression, the surface is a plane and IN always equals zero. For nonlinear regression, IN increases as the curvature of the expectation surface increases, invalidating the linear estimation procedure. Large IN values indicate unacceptable deviation from the tangent plane assumption. Parameter-effects curvature, PE, measures the lack of uniformity of the parameter lines on the tangent plane. In linear regression, parameter contours are parallel and equally-spaced when projected onto the tangent plane. The PE value of maximum relative curvature is obtained from a scaled version of the Hessian, i.e., the second derivatives of the model with respect to each parameter. For the linear case, all second derivatives equal zero resulting in a PE of zero. For nonlinear regression, a higher PE indicates higher degree of departure from the assumption of parallelism. The square root of the average squared curvature can be standardized by multiplying by $\mathrm{F}_{.05}$, p, n-p (Bates and Watts, 1980). When the standardized curvature measure is less than or equal to 0.4 , the curvatures are considered acceptable.

Ratkowsky (1990) suggests examining the close-to-linear behavior of each parameter. The measures used in this paper are Box's approximate measure of bias (1972), percent excess variance based on Lowry and Morton's asymmetry measure (1983) and Hougaard's approximate measure of skewness (1985). Using the under $1 \%$ rule-of-thumb for absolute \%bias suggests all parameters appear to have close-to-linear behavior. Lowry and Morton's asymmetry measure can be expressed as percentage of excess variance. Ratkowsky (quoted in Seber and Wild, 1989, p 188) gives reference values for the measure. Expressed as percentage, values $<1 \%$ indicate behavior is very close to linear: values between $1 \%$ and $5 \%$ indicate increasing skewness in the distribution of the parameter: and values $>5 \%$, skewness is very apparent in simulation. Skewness measures departure from symmetry. Hougaard's approximate skewness works well when $n>150$. Ratkowsky (1983) gives reference values for skewness. They are: $\mid$ skewness $\mid<0.1$ indicates behavior is very close to linear; between 0.1 and 0.25 , reasonably close to linear; between 0.25 and 1 , apparent skewness; and $>1$, considerable skewness. 


\section{Bi-logistic versus Segmented Bi-Logistic Model}

\section{RESULTS}

Figure 1 and Table 2 show how well the segmented model fits the data (146 min) from Steer 445 on day 2. The observed values of tmax and max Tb are $36.7 \mathrm{~min}$ and $1.89 \mathrm{C}$, respectively. The model predictions for these parameters are $36.8 \mathrm{~min}$ and $1.93 \mathrm{C}$, respectively. In other words, segmented model predicts tmax and max Tb very well (although max Tb appears slightly over estimated by 0.04). The mean square error, MSE of 0.0006 and standard error of the estimates, SEs, are small. The $95 \%$ confidence intervals, CIs, are adequate and exclude zero. Figure 2 and Table 3, on the other hand, show how well the bi-logistic model fits the same data. The MSE is greater (0.002) than that given by the segmented model. In addition, the max Tb is underestimated by $0.05(1.84 \mathrm{C})$ and predicted to occur 5.7 minutes later $(42.4 \mathrm{~min})$ than the observed time to maximum. So, in this particular case the segmented bi-logistic parameter estimates are closer to the observed values.

We present another example where the max Tb prediction is even better. Figure 3 and Table 4 show how well the segmented model fits the data $(78 \mathrm{~min})$ from Steer 336 on day 2. The observed estimates of tmax and max $\mathrm{Tb}$ are $33.0 \mathrm{~min}$ and $0.98 \mathrm{C}$, respectively. The model predictions for these parameters (33.50 min and $0.98 \mathrm{C}$, respectively) are very close to the observed values. The MSE equals 0.0005 and the SEs are small. The CIs are adequate and exclude zero. Figure 4 and Table 5, on the other hand, show the bi-logistic model provides a poorer fit to the same data. The MSE is larger (0.001). Tmax and max Tb are again underestimated (32.7 min and $0.92 \mathrm{C}$, respectively).

When the nonlinear behavior of the segmented model was assessed, the planarity assumption appeared acceptable for both segments (challenge and recovery) since INs are 0.017 and 0.042 , respectively. The parameter-effects curvature (PE) is also acceptable for both segments (0.124 and 0.128 , respectively). The bi-logistic model also appeared acceptable ( $\mathrm{IN}=0.046$ and $\mathrm{PE}=0.3385$ ). As for the measures proposed by Ratkowsky, the skewness, \%bias, and \%excess variance of Table 6 show that all parameters appear to have close-to-linear behavior for the segmented model. But, the asymptotic properties of the bi-logistic model (Table 7) indicate a problem with $\kappa_{2}$ (skewness $\left.=-.8527\right)$. The other parameters have close-to-linear behavior.

\section{Different recovery durations}

Both models (Eqs. [1] and [2]) were analyzed using three different times to estimate the recovery time $(86,146$, and $204 \mathrm{~min}$.) for Steer 445 on day 2 . When the segmented model was used (Figure 5), the maximum body temperature and the time to reach it were robust, decreasing slightly as the record length increased (max $\mathrm{Tb}$ from 1.91 to 1.90 and tmax from 36.3 to 35.7). However, when the bi-logistic model was used, the range for these two parameters was greater (max Tb from 1.89 to 1.79 and tmax from 41.8 to 42.7). Table 8 gives the parameter estimates from different recovery durations, and the \%change in the estimates with respect to the estimates from $146 \mathrm{~min}$. The results show that the segmented model was more robust to changes in recovery durations. Only one parameter, $\kappa_{2}$, had a \%change greater 
than $4 \%$. Whereas, the bi-logistic model had a \%change greater than $4 \%$ for four parameters

$\left(\tau_{1}, \delta, \kappa_{2}\right.$, and $\left.\tau_{2}\right)$. These large \%changes are highlighted in Table 8 .

\section{Hormetic Behavior}

The result associated with the fit of Eq. 3 is plotted in Figure 6 and tabulated in Table 9 for Steer 445 on day 2 . The estimate of $\gamma(-0.008)$ is significantly different from zero. The estimated $\max \mathrm{Tb}(1.90)$ is very close to the observed value (1.89) but tmax is predicted to occur 4.5 minutes later than the observed value. The MSE (0.0008) is slightly larger than that of segmented model (0.0006); and, although the planarity assumption appears acceptable (IN = $0.07)$, the uniform coordinates assumption does not $(\mathrm{PE}=1.04)$. In addition, the rate of recovery, $\kappa_{2}$, has a \%bias of 1.30 , \%excess variance of 1.60 and skewness of -5.31 . Therefore, a separate adjustment for the initial rate of increase in Tb during the challenge does not enhance the suitability of the model compared to the segmented version.

\section{SUMMARY}

The segmented version of the bi-logistic appeared superior to both the bi-logistic and hormetic models for modeling heat stress in processing feedlot cattle. The nonlinear curvatures validated the assumptions (i.e. both intrinsic and parameter-effects curvature are $<0.4$ ) and all parameter estimates showed close-to-linear behavior (i.e. \%bias, \%excess variance, and skewness criteria are satisfied). The segmented bi-logistic model followed the dynamics of the handling process. The predictions for time to reach maximum body temperature from the segmented bi-logistic model were closer to the observed value than either the bi-logistic or hormetic estimates. Moreover, the segmented bi-logistic model was robust to different definitions of recovery time.

\section{REFERENCES}

Bates, D. M. and D. L. Watts. 1980. "Relative curvature measures of nonlinearity." J. R. Statist. Soc. Ser. B. 42:1-25.

Box, M. J. 1972. “Bias in nonlinear estimation.” J. R. Statist. Soc. Ser. B. 33:171-201.

Hougaard, P. 1985. "The appropriateness of the asymptotic distribution in a nonlinear regression model." J. R. Statist. Soc. Ser. B. 47:103-114.

Lowry, R. and R. Morton. 1983. "An asymmetry measure for estimators in non-linear regression models." Proc. $44^{\text {th }}$ Session Int. Statist. Inst., Madrid, Contributed Papers $1: 351-354$.

Parkhurst, A. M. and T. L. Mader. 2000. "Using nonlinear growth curves to estimate heat stress in processing feedlot cattle." Twelfth Annual Kansas State University Conference on Applied Statistics in Agriculture. Manhattan, Kansas.

Ratkowsky, D. 1990. Handbook of nonlinear regression models. Marcel Dekker, New York and Basel. 
SAS. 1999. SAS/STAT User' s Guide. Version 8. SAS Institute Inc., Cary, NC.

Seber, G. and C. Wild. 1989. Nonlinear regression. John Wiley \& Sons, New York.

Schabenberger, O. and J. B. Birch. 2001. "Statistical dose-response models with hormetic effects. Human and Ecological Risk Assessment" 7(4): 891-908. 
Table 1. Experimental Design

\begin{tabular}{|l|l|l|l|l|l|l|l|}
\hline & \multirow{2}{*}{ Steer } & Day 1 & $\begin{array}{l}\text { Day } \\
\mathbf{2}\end{array}$ & $\begin{array}{l}\text { Day } \\
\mathbf{3}\end{array}$ & $\begin{array}{l}\text { Days } \\
\mathbf{4 \& 5}\end{array}$ & $\begin{array}{l}\text { Day } \\
\mathbf{6}\end{array}$ & $\begin{array}{l}\text { Day } \\
\mathbf{7}\end{array}$ \\
\cline { 3 - 8 } & & \multicolumn{7}{|c|}{ Distance (ft) } \\
\hline \multirow{2}{*}{ Pen 1 } & 377 & & 1200 & 1200 & & 200 & 200 \\
\cline { 2 - 7 } & 445 & & 1200 & 1200 & & 200 & 200 \\
\hline Pen 2 & 442 & & 200 & 200 & & 1200 & 1200 \\
\hline & 508 & & 200 & 200 & & 1200 & 1200 \\
\hline
\end{tabular}

Figure 1. Segmented bi-logistic model (Eq. 2) Figure 2. Bi-logistic model (Eq. 1)

Steer 445 on Day 2 (146 min)

Observed tmax $=36.7$ Max $\mathrm{Tb}=1.89$
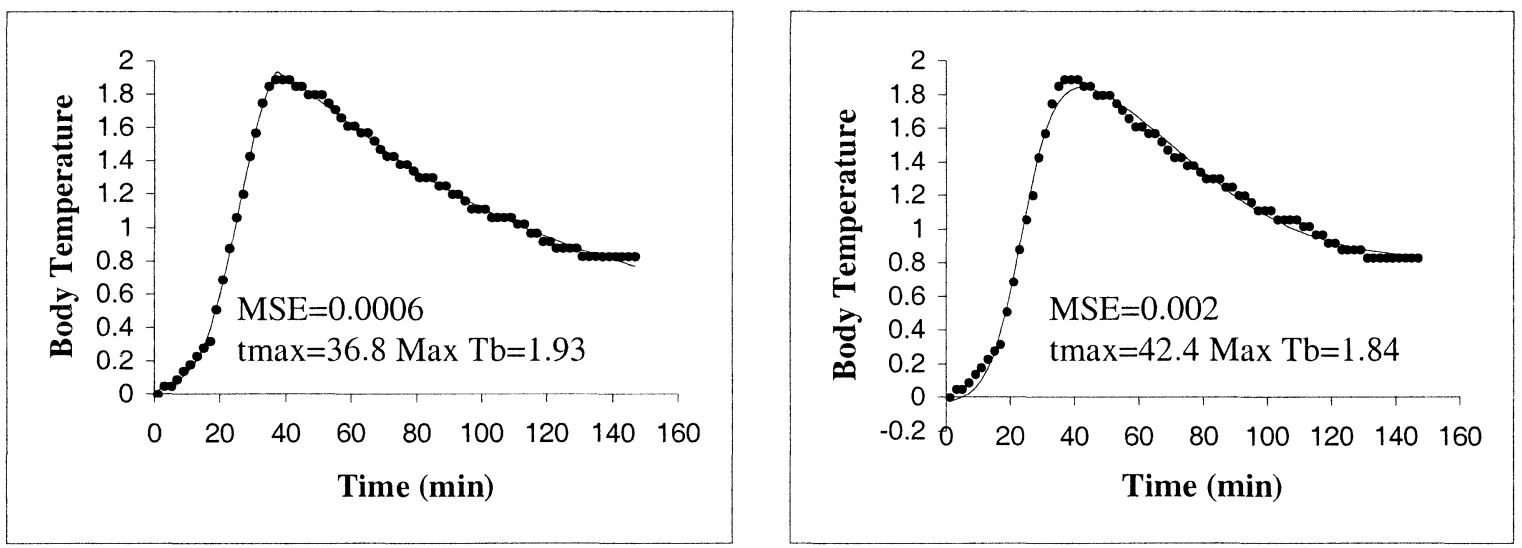

Table 2. Segmented model (Steer 445 on Day 2, $146 \mathrm{~min}$ ), MSE=0.0006

\begin{tabular}{|l|r|r|r|r|}
\hline Parameter & \multicolumn{1}{l|}{ Estimate } & \multicolumn{1}{l|}{ SE } & Lower CI & Upper CI \\
\hline$\alpha$ & 2.187 & 0.057 & 2.072 & 2.302 \\
\hline$\kappa_{1}$ & -0.178 & 0.006 & -0.190 & -0.167 \\
\hline$\tau_{1}$ & 25.538 & 0.381 & 24.778 & 26.299 \\
\hline$\tau_{2}$ & 117.2 & 0.891 & 115.4 & 119.0 \\
\hline$\kappa_{2}$ & -0.0490 & 0.0036 & -0.0561 & -0.0418 \\
\hline
\end{tabular}

Table 3. Bi-logistic model (Steer 445 on Day 2, $146 \mathrm{~min}$ ), MSE=0.002

\begin{tabular}{|l|r|r|r|r|}
\hline Parameter & Estimate & \multicolumn{1}{|l|}{ SE } & Lower CI & Upper CI \\
\hline$\alpha$ & 2.200 & 0.104 & 1.992 & 2.407 \\
\hline$\kappa_{1}$ & -0.191 & 0.012 & -0.214 & -0.167 \\
\hline$\tau_{1}$ & 23.631 & 0.211 & 23.209 & 24.052 \\
\hline$\delta$ & 0.803 & 0.021 & 0.761 & 0.846 \\
\hline$\kappa_{2}$ & -0.047 & 0.004 & -0.055 & -0.039 \\
\hline$\tau_{2}$ & 70.110 & 3.206 & 63.714 & 76.507 \\
\hline
\end{tabular}


Figure 3. Segmented bi-logistic model (Eq. 2) Figure 4. Bi-logistic model (Eq. 1)

Steer 336 on Day 2 (78 min)

Observed tmax $=33$ Max $\mathrm{Tb}=0.98$
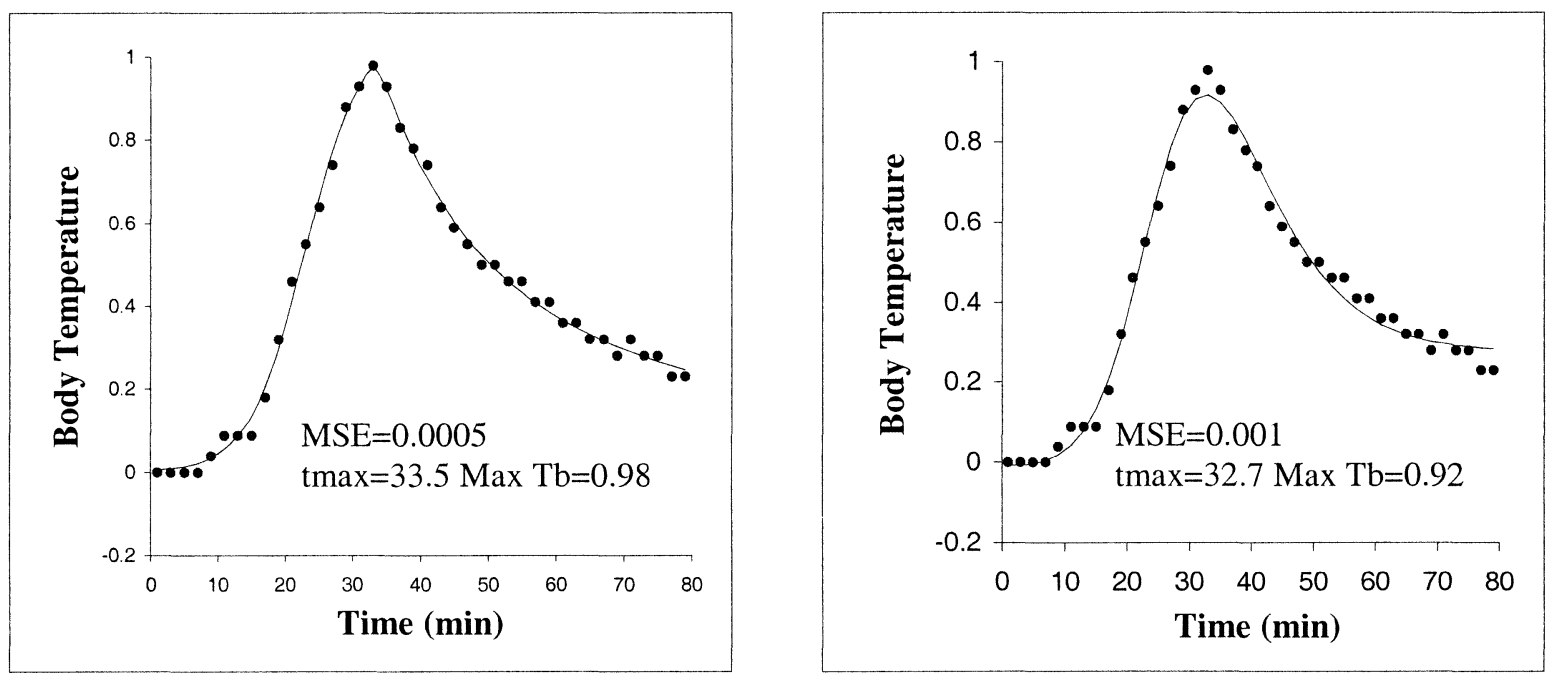

Table 4. Segmented model (Steer 336 on Day 2, $78 \mathrm{~min}$ )

\begin{tabular}{|l|r|r|r|r|}
\hline Parameter & \multicolumn{1}{|c|}{ Estimate } & \multicolumn{1}{|l|}{ SE } & Lower CI & Upper CI \\
\hline$\alpha$ & 1.053 & 0.031 & 0.989 & 1.117 \\
\hline$\kappa_{1}$ & -0.244 & 0.014 & -0.271 & -0.216 \\
\hline$\tau_{1}$ & 22.787 & 0.364 & 22.047 & 23.528 \\
\hline$\kappa_{2}$ & -0.0727 & 0.0120 & -0.0971 & -0.0483 \\
\hline$\tau_{2}$ & 50.981 & 0.694 & 49.571 & 52.391 \\
\hline
\end{tabular}

Table 5. Bi-logistic model (Steer 336 on Day 2, 78 min)

\begin{tabular}{|l|r|r|r|r|}
\hline Parameter & Estimate & \multicolumn{1}{|l|}{ SE } & Lower CI & Upper CI \\
\hline$\alpha$ & 1.815 & 0.758 & 0.274 & 3.356 \\
\hline$\kappa_{1}$ & -0.206 & 0.038 & -0.283 & -0.128 \\
\hline$\tau_{1}$ & 23.739 & 0.982 & 21.743 & 25.735 \\
\hline$\delta$ & 0.275 & 0.014 & 0.246 & 0.303 \\
\hline$\kappa_{2}$ & -0.119 & 0.008 & -0.135 & -0.103 \\
\hline$\tau_{2}$ & 35.375 & 4.918 & 25.380 & 45.370 \\
\hline
\end{tabular}


Table 6. The estimates of parameters and their asymptotic properties for challenge and recovery (Steer 445 on Day 2) for the segmented bi-logistic model

\begin{tabular}{|c|r|r|l|l|l|l|r|r|r|}
\hline \multicolumn{9}{|c|}{ Challenge } & \multicolumn{5}{|c|}{ Recovery } \\
\hline $\begin{array}{l}\text { Para- } \\
\text { meter }\end{array}$ & Estimate & $\begin{array}{l}\text { Skew- } \\
\text { ness }\end{array}$ & $\begin{array}{l}\text { Bias } \\
(\%)\end{array}$ & $\begin{array}{l}\text { Excess } \\
\text { Var(\%) }\end{array}$ & $\begin{array}{l}\text { Para- } \\
\text { meter }\end{array}$ & Estimate & $\begin{array}{l}\text { Skew- } \\
\text { ness }\end{array}$ & $\begin{array}{l}\text { Bias } \\
(\%)\end{array}$ & $\begin{array}{l}\text { Excess } \\
\text { Var(\%) }\end{array}$ \\
\hline$\alpha$ & 2.148 & 0.237 & 0.087 & 0.430 & tmax & 38.247 & -0.196 & -0.078 & 0.669 \\
\hline$\kappa_{1}$ & -0.181 & 0.096 & 0.083 & 0.121 & $\kappa_{2}$ & -0.0538 & -0.0843 & 0.177 & 0.285 \\
\hline$\tau_{1}$ & 25.299 & 0.198 & 0.043 & 0.373 & $\tau_{2}$ & 117.2 & 0.0567 & 0.006 & 0.051 \\
\hline
\end{tabular}

Table 7. Asymptotic properties of the bi-logistic model (Steer 445 on Day 2)

\begin{tabular}{|c|r|r|r|r|}
\hline Parameter & Estimate & Skewness & Bias (\%) & $\begin{array}{c}\text { Excess } \\
\text { Var(\%) }\end{array}$ \\
\hline$\alpha$ & 2.1998 & 0.2835 & 0.0143 & 0.0797 \\
\hline$\kappa_{1}$ & -0.1906 & -0.1257 & 0.0361 & 0.0221 \\
\hline$\tau_{1}$ & 23.6308 & 0.0105 & -0.0145 & 0.1378 \\
\hline$\delta$ & 0.8031 & -0.0283 & -0.0640 & 0.0908 \\
\hline$\kappa_{2}$ & -0.0474 & -0.8527 & 0.1673 & 0.2516 \\
\hline$\tau_{2}$ & 70.1103 & -0.2197 & 0.0163 & 0.1297 \\
\hline
\end{tabular}

Figure 5. Different recovery durations for the segmented bi-logistic model (Eq. 2)

\section{Steer 445 on Day 2}

\section{Observed tmax=36.7 Max $\mathrm{Tb}=1.89$}
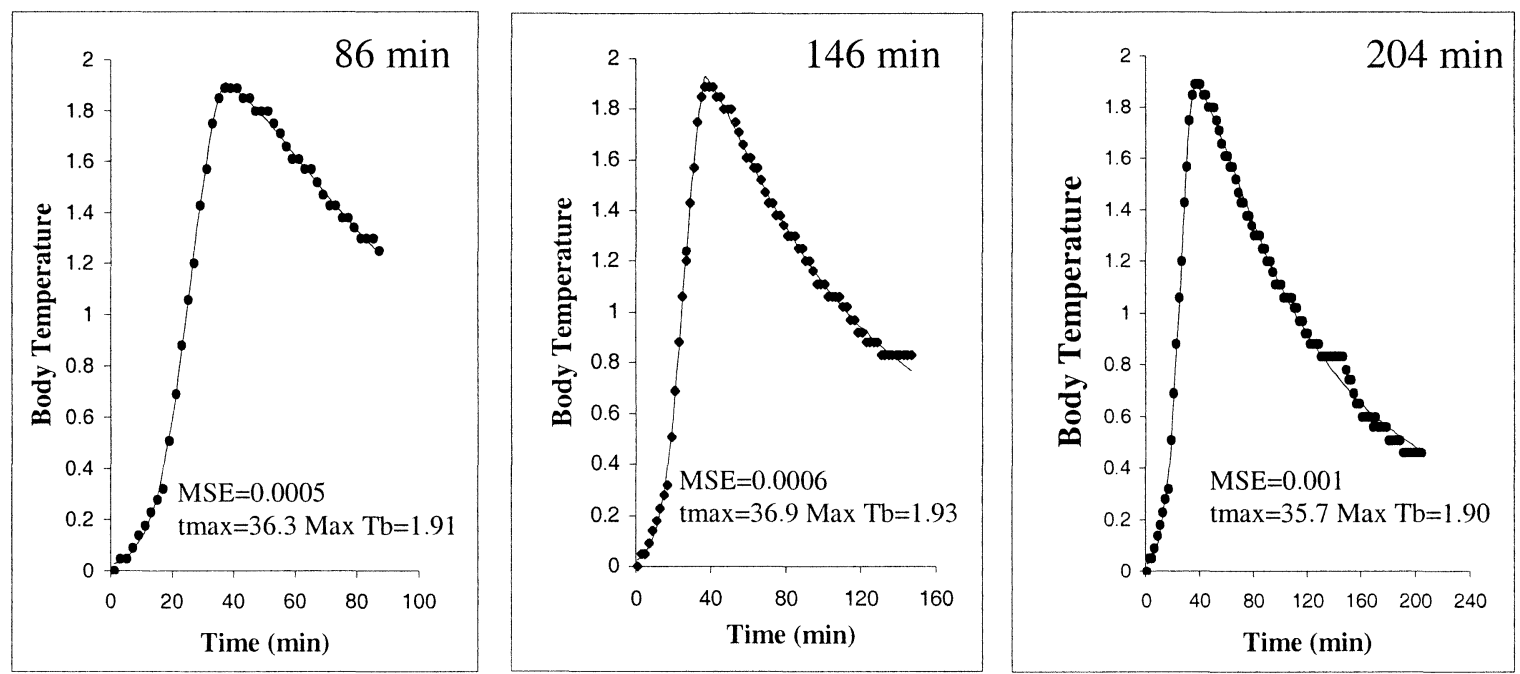
Table 8. Different recovery durations (Steer 445 on Day 2)

\begin{tabular}{|l|c|r|r|r|r|r|}
\hline & Para- & \multicolumn{2}{|c|}{ 86 min } & 146 min & \multicolumn{2}{|c|}{ 204 min } \\
\cline { 2 - 7 } & meter & Estimate & \% Change & Estimate & Estimate & \% Change \\
\hline \multirow{3}{*}{$\begin{array}{l}\text { Segmented } \\
\text { Bi-logistic }\end{array}$} & $\alpha$ & 2.203 & 0.732 & 2.187 & 2.224 & 1.692 \\
\cline { 2 - 7 } Model & $\kappa_{1}$ & -0.177 & -0.562 & -0.178 & -0.176 & -1.124 \\
\cline { 2 - 7 } & $\tau_{1}$ & 25.634 & 0.376 & 25.538 & 25.757 & 0.858 \\
\cline { 2 - 7 } & $\kappa_{2}$ & -0.033 & $\mathbf{- 3 2 . 6 5 3}$ & -0.049 & -0.031 & $\mathbf{- 3 6 . 7 3 5}$ \\
\cline { 2 - 7 } & $\tau_{2}$ & 112.9 & -3.669 & 117.2 & 116.9 & -0.256 \\
\cline { 2 - 7 } & $\mathrm{tmax}$ & 36.266 & -1.571 & 36.845 & 35.707 & -3.089 \\
\hline \multirow{4}{*}{$\begin{array}{l}\text { Mi-logistic } \\
\text { Model }\end{array}$} & $\alpha_{1}$ & 2.255 & 2.500 & 2.200 & 2.210 & 0.450 \\
\cline { 2 - 7 } & $\kappa_{1}$ & -0.186 & -2.204 & -0.191 & -0.186 & -2.361 \\
\cline { 2 - 7 } & $\tau_{1}$ & 24.736 & $\mathbf{4 . 6 7 6}$ & 23.631 & 22.465 & $\mathbf{- 4 . 9 3 2}$ \\
\cline { 2 - 7 } & $\delta$ & 1.214 & $\mathbf{5 1 . 1 1 4}$ & 0.803 & 0.472 & $\mathbf{- 4 1 . 2 5 3}$ \\
\cline { 2 - 7 } & $\kappa_{2}$ & -0.081 & $\mathbf{7 0 . 0 4 2}$ & -0.047 & -0.031 & $\mathbf{- 3 4 . 5 9 9}$ \\
\cline { 2 - 7 } & $\tau_{2}$ & 54.533 & $\mathbf{- 2 2 . 2 1 9}$ & 70.110 & 84.630 & $\mathbf{2 0 . 7 0 9}$ \\
\hline
\end{tabular}

Figure 6. Hormetic behavior (Eq. 3)

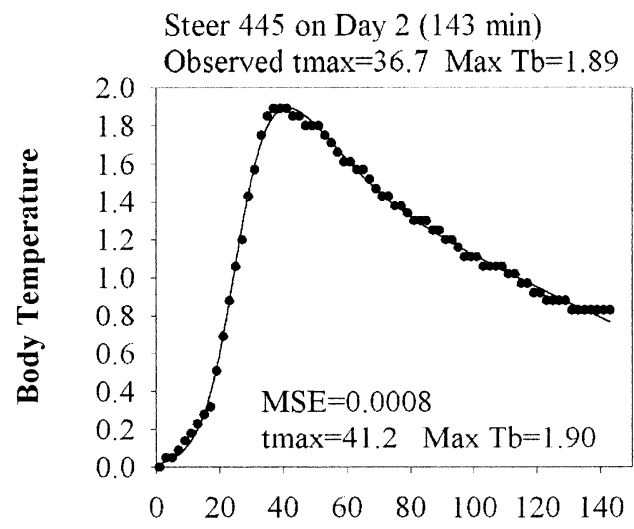

Time (min)

Table 9. Hormetic behavior (Steer 445 on Day 2)

\begin{tabular}{|l|r|r|r|r|r|r|r|}
\hline Parameter & Estimate & \multicolumn{1}{l|}{ SE } & Lower CI & Upper CI & Bias (\%) & \multicolumn{1}{l|}{$\begin{array}{l}\text { Excess } \\
\text { Var (\%) }\end{array}$} & \multicolumn{1}{l|}{ Skewness } \\
\hline$\alpha$ & 2.311 & 0.289 & 1.733 & 2.889 & -0.326 & 0.4218 & -0.569 \\
\hline$\kappa_{1}$ & -0.185 & 0.015 & -0.215 & -0.156 & 0.144 & 0.208 & -0.717 \\
\hline$\tau_{1}$ & 24.927 & 0.329 & 24.271 & 25.584 & -0.036 & 0.307 & -1.005 \\
\hline$\delta$ & 1.896 & 0.056 & 1.784 & 2.008 & -0.0538 & 0.305 & 0.700 \\
\hline$\kappa_{2}$ & -0.082 & 0.016 & -0.114 & -0.050 & 1.304 & 1.601 & -5.319 \\
\hline$\tau_{2}$ & 45.113 & 7.025 & 31.083 & 59.143 & 0.512 & 0.456 & 1.416 \\
\hline$\gamma$ & -0.008 & 0.0004 & -0.009 & -0.007 & -0.0867 & 0.264 & -0.533 \\
\hline
\end{tabular}

